\title{
Research and Application of Growth Management Model of Tree
}

\author{
Liying Duan, Junling Li, Qian Dong, Qingyong Jin, Xuning Liu \\ Department of Computer, Shijiazhuang University, Shijiazhuang, Hebei,China \\ sjzhei@163.com
}

Keywords: tree, growth management, model

\begin{abstract}
With the application of growth model and computer techniques in tree growth management, in order to grasp growth pattern of tree, further improve the level of tree growth management, and make the research on the tree growth model gradually develop towards the intelligent and science. The paper carried out simulation research technology for management model of the tree growth, meanwhile the database technology and modeling technology are used in the tree growth model, so the management model of tree growth is established, then visually display the states which are at different stages of tree growth management, which can contribute to predict the development states of tree growth. The research on tree growth model has important theory and practical significance in understanding the yield of the various parts of the tree and in taking the reasonable management measures.
\end{abstract}

\section{Introduction}

Tree growth simulation model is to regard the tree and the environmental factors as a whole, and the principles and methods of systems analysis are used. The theories and research findings which considers tree physiology, ecology, agronomy, agro-meteorology and other disciplines are integrated to carry out quantitative analysis on the physiological process such as growth development and yield formation of tree and the experimental data of their relationship with environmental factors, and establish corresponding mathematical models, and then simulation study of dynamic quantitative analysis and tree growth process are made on the computer. The tree growth model can not only simulate the final yield and quality of trees, but also display the occurrence of tree physiological and ecological process and evolutionary dynamics during the reproductive process of tree growth[1].

The development of tree growth management model will make comprehensive use of information management, automatic monitoring, dynamic simulation, virtual reality, knowledge engineering, precise control and other modern information technologies, information and digital technology in production factors and production process of tree are regarded as the main research targets, and develop information management of tree growth and resources management, automatic monitoring of tree growth management state, the digital simulation of tree growth management process, tree growth management knowledge expression modeling, visual design of key technologies in tree growth management and so on, further study the hardware and software technology of tree growth management development, then achieving digital control, high precision, visualization, and network of prediction and management of tree growth management model[2].

\section{Key Technologies}

Growth Model. The growth and development process of tree are very complex, and the factors are the following: First, the control variables and impact factors are too much; Each variable and growth results may have strong dynamic coupling changing process; The growth process presents random, non-linear variability and mutation. In fact, it is very difficult to establish tree growth model which fully reflects the above characters. In general, under certain assumptions and according to some specific research on the trees, then the mathematical treatment in growth model of tree is performed. 
Investigation and analysis of relevant data on tree are performed, we can get the following requirement of tree growth model: The tree resource behalf data are used, the optimal mathematical algorithms are performed to establish the optimal tree growth model, which is able to find and call the existing tree growth models, according to the existing survey data of tree resources we can predict future growth states of tree, and the form of data are stored for the statistics, analysis and output; The growth models of incorrect tree are modified or deleted, therefore generate the new growth model that meets the growth conditions[3].

Resource model of tree growth management is responsible for the construction, query and information management of new model, and the model construction can generate based on the existing modified model, add directly a new model system, the system provides ways to connect the survey data of the actual tree resources and generate a new model, the user can build a good new model in the deposit model. The existing tree growth models and algorithm components are combined to build the tree growth reproductive system, and the integrated simulation system is made, finally the large number of experimental data are used to make testing and inspection of model, and the methods of trial and error are used to adjust the model parameters to simulate the value, so the agreement value of observed value and the best value is regarded as final parameters[4].

Database of Tree Growth Management Resource. The tree growth management resource database supports the query from the tree growth model and connects with the existing data to calculate the new data, which are used to predict or update data. The tree growth management resources database management system includes the management and maintenance functions of the resource base of tree growth management, then including tree growth and management of resources survey data searching, data management, system maintenance and so on. In order to meet the growth of agricultural trees, the management parameters of various trees growth not only timely understand and grasp, but also summarize the laws of tree growth and the requirements of the various parameters[5], the tree growth management resource database can give trees growth and management of resources real-time and the display of historical data table and the curve of real-time trend curves and historical trends, which can ensure that users can look at real-time resource parameters of the tree growth of the current value and trend curve[6].

Visualization Model. Visual model can call the graphic symbols that represent the various organs, and graphic symbols are linked to computer animation of the tree in accordance with the topology, so generate the two-dimensional or three-dimensional graphics and growth process. Visual model of the tree consists of a number of sub-model such as the geometry light, texture and rendering[7].

The main contents of the tree visualization also include fast rendering of graphics, the bending of branches, tree phototropism and geotaxis etc. The visualization technology of trees will transform the data that constitutes tree morphological structure of into the form of graphics or images on the computer simulation techniques. The tree structure is a description of method and geometry parameters inversion of specific tree spatial form, which includes the following: Trees are drawn by means of light, texture, rendering. However, due to the complexity of the structure of the trees, such as self-similar characters, multi-branch branches, branches of the natural bending of the phototropism of trees, the branches of trees, smooth and non-smooth connection, variability and the directly or indirect impact of environmental conditions on the leaves of trees, the three-dimensional visual simulation of the trees becomes very difficult[8].

Integration Technology. The combining methods of tree growth model, environmental prediction models and expert systems are considered, and the technology is embedded in the growth model system so as to achieve optimal control of tree environment. This method takes full advantage of the predictions mechanism of tree growth model, the experience of experts in agronomy, the leaps and bounds progress are compared with the present environmental control method. Considering the influence of many factors, and it is easy to control. In short, the three fundamental roles of the tree simulation research are interrelated and indivisible, and the roles are mainly following: 
- Understand the process or mechanism of tree growth, tree growth model is understood or explained in science, the scientific nature of model is much stronger, then understanding of the process or mechanism of tree growth that explain the role is much better, and is regarded as a measures of model science.

- The prediction role is basic requirement of the growth model, and is basic purpose of the tree simulation research, and judge the performance of comprehensive standard. Model prediction is based on the role of science, if science is not good, the accuracy of prediction is low.

- The roles of control are made, the ultimate goal of regulation of tree growth simulation is to carry out the necessary regulation and control on tree growth and production management, then makes the process of tree visually display at different stages of tree growth. Regulation of the tree model is further extension and development of prediction, and is the premise of prediction.

\section{Establishment of Tree Growth Model}

The establishment of virtual tree growth model requires the following steps:

- The conditions of tree growth are shown at the different stages of tree growth and under different environmental factors, we can judge growth mode of branch, the type of scores, then determine the overall framework to describe the tree morphology.

- Three-dimensional digital instruments are used to carry out continuous and accurate measurement on the topology and geometric features during the process of tree growth, then mathematical statistics or artificial intelligence methods are used to carry out analysis and processing of measured data and extract the structural rules of tree morphology.

- The appropriate modeling tools are chosen, and the help is from measured tree growth rules, then the topology model and the geometric structure model of trees are established

- The combination of the morphogenetic model and specific physiological and ecological model are made, which are based on biomass production and distribution in different organs at each stage, and calculate the growth and development state of trees in various stages of growth.

- Based on the geometric dimensions and spatial position of the various growth stages which are calculated for each tree organs, the visual model structure is used to build three-dimensional tree graphics; The computer graphics technology such as texture mapping and light calculation, the depth of blanking anti-aliasing, projection and so on are used to deal with trees in three-dimensional geometric structure to generate a realistic image of the trees graphics.

\section{Implementation and Test of Model}

Composition of Modules. The model system consists of the model management module, knowledge management module, inference engine, database management module, and model base, knowledge base, and human-computer interaction interface.

There exits close relationship among the model management modules, the database management module and knowledge base management module. Model management module is responsible for the coordination of the work between every models, then achieving the quantitative reasoning of different objectives. Database resources and user databases include the following: resource database are stored in trees and local resource data, such as pest and disease data, meteorological data and the user database are stored in the system data, such as input data in the operation of system, the obtained intermediate inference results and the final results. The database management module is responsible for coordination that the data are provided for knowledge base, there may have conflict due to the diversity of knowledge between the different knowledge, the knowledge management is used to carry out comparison, choices, refining in order to obtain reliable knowledge.

The inference engine searches and activates the appropriate knowledge from the knowledge base, according to the problems that user proposes new instances, or asks the user to obtain relevant facts by the reasoning and the known facts in the knowledge base, until the final problems are solved or the known conditions can not be solved so far. After the inference engine comes to conclusion, and the combination of external environmental factors is regarded as the input of the growth machine 
model, and the interaction of physiological and ecological model contribute to calculate the growth conditions of trees at various growth stages and under different environmental factors, and then the computer visualization techniques are used to generate three-dimensional graphics that reflects the current growth status of the tree or growth process of animation, and the visually display intelligent decision-making results of systems.

Test of Model. The test of model is to verify the suitable degree of simulation results and actual results, and check whether the model is applied in different environments by root mean square deviation between the observations and simulation values, the statistical agreement between simulation results and observations analysis and the relationship between the histogram are used to visually show agreement and reliability of analog respectively, it is said that the number of samples, the analog value, the value of the observations are much less, the prediction accuracy of the higher values and observed values of the average error are very good, then the consistency of simulation and actual values are very good, which indicate that the simulation results are generally greater than the analog value, and the actual deviation of simulated poor model will run in a simulated environment and is validated. After validation we can understand the simulation results are close to the real approximation of the model, thus confirming the correctness of the tolerance range.

\section{Summary}

The development of tree growth management expert systems and simulation models for the management of the modernization of tree growth and information provide new ways and means.. However the traditional agricultural expert system contains a lot of expertise and a strong regional and time parameters, and the low level of quantitative space-time adaptive differential does not have prediction ability, limiting the system decision-making under different environmental conditions, wide adaptability and accuracy.

Tree growth management simulation models have strong functions of system integration and dynamic prediction, the parameters of the model are required for high precision, poor operation, but also are difficult to direct the production system management decisions. System analysis methods and plant modeling techniques are used in knowledge expression system for tree growth, and it is expected to have both the advantages of tree simulation models and expert systems, and achieve the integration and coordination of dynamic prediction and management decision-making, so as to lay the foundation for the accurate and digitization of growth management of tree.

\section{References}

[1] Lindenmay A. Mathematical models for cellular interaction in development, Parts I andII. Journal of Theoretical Biology ,1968, 18:280-315.

[2] Smit h A R. Trees fractals and formal languages. Computer Graphics, 1984, 18(3) : 12-13.

[3] De Reffye P H, Edelin C, Francon J etal . Tree models faithful to botanical structure and development . Computer Graphics , 1988 , 22 (4) : 151-158.

[4] Oppendheimer P E. Real time design and animation of fractal trees and trees. Computer Graphics , 1986, 20(4) : 55-64.

[5] Barnsley M F , Demko S. Iterated function systems and global const ruction of fractals . R Soc London Ser, 1985 (A399) : 243-275.

[6] Reeves WT, Blau R. Approximate and probabilistic algorithms for shading and rendering structured particle systems[J] . Computer Graphics , 1985, 19 (3) : 313-322. 
[7] Hu B G,De P R , Zhao X, et al . GreenLab : A new methodology towards tree functional

[8] structural model-Structural aspect. Proceedings of 2003 International Symposium on Tree Growth Modeling, Simulation,Visualization and their Application. Beijing, Tsinghua University Press and Springer, $2003: 21235$.

[9] Huai Xiaoyong, Xiong Fanlun. Research on new architecture intelligent systems and its system development environment-visual XF6. 1. International Symposium on Intelligent Agriculture Information Technology, Beijing ,P. R. China , 2000 , 124. 